\title{
Coexisting vacua and effective gravity
}

\author{
F. R. Klinkhamer a, G. E. Volovik ${ }^{\mathrm{b}, \mathrm{c}}$ \\ ${ }^{a}$ Institute for Theoretical Physics, University of Karlsruhe (TH), \\ 76128 Karlsruhe, Germany \\ ${ }^{\mathrm{b}}$ Helsinki University of Technology, Low Temperature Laboratory, \\ P.O. Box 2200, FIN-02015 HUT, Finland \\ ${ }^{\mathrm{c}}$ Landau Institute for Theoretical Physics, 119334 Moscow, Russia
}

\begin{abstract}
Our Universe may be a domain separated by physical phase boundaries from other domain-Universes with different vacuum energy density and matter content. The coexistence of different quantum vacua is perhaps regulated by the exchange of global fermionic charges or by fermion zero modes on the phase boundary. An example would be a static de-Sitter Universe embedded in an asymptotically flat spacetime.
\end{abstract}

Key words: general relativity, cosmology, interface between quantum vacua PACS: 04.20.Cv, 98.80.Hw, 05.70.Np

\section{Introduction}

Condensed-matter systems provide several examples of emerging quantum fields (see [1-3] and references therein). Effective gravity appears in the lowenergy corner of condensed matter either via the metric $g_{\mu \nu}(\mathbf{r}, t)$ or via the vierbeins $e_{\mu}^{a}(\mathbf{r}, t)$. These fields are functions of the coordinates $\mathbf{r}$ and $t$ of the underlying flat Galilei or Minkowski spacetime, just as the other emerging fields (e.g., fermionic and gauge fields).

Email addresses: frans.klinkhamer@physik.uni-karlsruhe.de (F. R. Klinkhamer), volovik@boojum.hut.fi (G. E. Volovik). 
Emergent gravity does not require an a-priori notion of curved spacetime. The latter is a secondary phenomenon experienced by low-energy quasiparticles, whose dynamics in the geometric-optics limit can be described in terms of motion along the geodesic curves provided by the effective metric $g_{\mu \nu}(\mathbf{r}, t)$. For the 'poor physicist' of Ref. [3] who can only use quasiparticles with energies low compared to the corresponding cut-off $E_{\text {Planck }}$, the world is curved.

The most interesting condensed-matter models have effective gravity emerging together with non-Abelian gauge fields and Weyl fermions in systems with Fermi points (generic points of level crossing at zero energy) [1]. It may well be (though this has not yet been proven) that, if the hierarchy of energy scales is favorable, the dynamics of the effective gravity is described by an Einstein-Hilbert action emerging at low energy. For the 'poor physicist' living in this particular type of condensed matter, any connection to the 'fundamental' underlying spacetime is lost and effective gravity is (at least, locally) indistinguishable from a fundamental interaction.

These considerations force us to pay more attention to approaches which consider the gravitational field as a conventional non-Abelian spin-2 field in Minkowski spacetime [4]. But, even if these theories in the low-energy corner lead to the same local equations as Einstein gravity, the global structure of the Universe can be different. The latter can, in principle, be detected by following the particle trajectories [5].

Moreover, the mere existence of fundamental coordinates suggests that in some cases the so-called coordinate singularities, which appear in certain solutions of the Einstein equations, are not gauge artifacts but real physical singularities. This occurs if the coordinate transformation needed to remove the singularity belongs to a class of transformations forbidden in the global fundamental spacetime.

One example may be provided by a static Einstein universe with positive cosmological constant $\Lambda_{0}[6]$, whose line element can be written as

$$
d s^{2}=-d t^{2}+\frac{d r^{2}}{1-r^{2} / R_{0}^{2}}+r^{2}\left(d \theta^{2}+\sin ^{2} \theta d \phi^{2}\right)
$$

Here, we have used relativistic units so that the velocity of light in "empty" space has the value $c=1$ (later, we also set $\hbar=1$ ). In the original Einstein theory, space is the three-dimensional (3D) compact hypersurface of the 4D spacetime $\mathbb{R} \times S^{3}$. This Einstein Universe is geodesically complete and free from singularities, which means that the coordinate singularity at $r=R_{0}$ is a gauge artifact caused by the specific choice of coordinates on the compact space $S^{3}$. More precisely, the space described by the metric (1) for $r \in\left[0, R_{0}\right)$ 
is only one hemisphere of $S^{3}$, whereas the whole space of the original Einstein Universe has $r=R_{0} \sin \Psi$ for polar angle $\Psi \in[0, \pi]$; cf. Chap. XII, Sec. 133 of Ref. [7].

Let us now consider the Universe (1) from the point of view of gravity as a field determined in Minkowski spacetime $M^{4}$. Since $M^{4}$ is infinite, the finite Einstein Universe must be a part of the infinite system. One option is to replace the coordinate singularity at $r=R_{0}$ with a real singularity (or, better, a physical boundary between two regions) at $r=R \leq R_{0}$. The Einstein Universe lies inside the boundary (interface) and the rest of Minkowski spacetime outside:

$$
\begin{aligned}
& d s^{2}=-d t^{2}+\frac{d r^{2}}{1-r^{2} / R_{0}^{2}}+r^{2}\left(d \theta^{2}+\sin ^{2} \theta d \phi^{2}\right), \quad r<R \leq R_{0}, \\
& d s^{2}=-d t^{2}+d r^{2}+r^{2}\left(d \theta^{2}+\sin ^{2} \theta d \phi^{2}\right), \quad r>R
\end{aligned}
$$

with constants $R$ and $R_{0}$ to be determined later. Observe that this metric describes a static spacetime with an inhomogeneous spatial section: a Universe with constant matter density and cosmological constant $\Lambda=\Lambda_{0}>0$ inside, and an empty flat space with $\Lambda=0$ outside. In contrast, the original Einstein Universe has a homogeneous spatial section, $S^{3}$ being a symmetric space. Indeed, the "Universe" from Eqs. (2ab) does have a center (i.e., a point with equal distance to the interface in each direction), even though this may not be apparent to the 'poor physicist' living deep inside. For this observer, the inside region serves as a static Einstein Universe.

The metric (2a) obeys the Einstein equations, with matter density $\rho_{\mathrm{m}}$, cosmological constant $\Lambda_{0}=\rho_{\mathrm{vac}}$, and horizon radius $R_{0}$ related by the following equations:

$$
\Lambda_{0}=\rho_{\mathrm{vac}}=\frac{1}{2} \rho_{\mathrm{m}}(1+3 w), \quad \frac{1}{4 \pi G R_{0}^{2}}=\rho_{\mathrm{m}}(1+w)
$$

where $P_{\mathrm{m}}=w \rho_{\mathrm{m}}$ is the matter equation of state. The total energy of the Einstein Universe including the interface $(r \leq R)$ must be zero. This then matches the Minkowski metric of the outer region $(r>R)$, where matter is absent and the cosmological constant is zero, $\rho_{\mathrm{m}}=\rho_{\mathrm{vac}}=0$. The outer region exerts no pressure on the inner region, which behaves as if there were no external environment.

However, the discontinuity of the metric at $r=R$ and the jump in the vacuum energy demonstrate that the singularity is physical. The jump in vacuum energy requires a surface layer which separates the "true" vacuum outside $\left(\rho_{\mathrm{vac}}=0\right.$ for $\left.r>R\right)$ from the "false" vacuum inside $\left(\rho_{\mathrm{vac}}=\Lambda_{0}>0\right.$ for $\left.r<R\right)$. 
Within the surface layer, the original theory is modified. In this approach, the Einstein Universe represents a bubble of false vacuum embedded in true vacuum. Note also that the additional pressure from the surface tension and the gravitational effects of the massive boundary modify the balance between the outer and inner vacua.

Another example of a false-vacuum bubble is a de-Sitter Universe [8] embedded in a space-time which is flat at spatial infinity,

$$
\begin{aligned}
& d s^{2}=-\left(1-r^{2} / R_{0}^{2}\right) d t^{2}+\frac{d r^{2}}{1-r^{2} / R_{0}^{2}}+r^{2} d^{2} \Omega, \quad r<R \leq R_{0}, \\
& d s^{2}=-(1-2 G M / r) d t^{2}+\frac{d r^{2}}{1-2 G M / r}+r^{2} d^{2} \Omega, \quad r>R,
\end{aligned}
$$

with solid angle element $d^{2} \Omega \equiv d \theta^{2}+\sin ^{2} \theta d \phi^{2}$, de-Sitter length scale [7]

$$
R_{0} \equiv \sqrt{3 /\left(8 \pi G \Lambda_{0}\right)},
$$

and constants $R$ and $G M$ to be determined later. Note that, historically, Weyl [9] appears to have been the first to try to replace the de-Sitter horizon at $r=R_{0}$ by a physical surface layer and glue together two static de-Sitter Universes.

The embedding (4ab) also has a condensed-matter analogy; see Ref. [10] for a review. This was originally discussed in relation to the event horizon of a Schwarzschild black hole by Chapline et al. [11], and later by Mazur and Mottola [12] and by Dymnikova and Galaktionov [13], who viewed the eventhorizon coordinate singularity as a physical singularity. In their treatment, the horizon is again a massive surface layer. Similar junctions between different space-times have been considered for inflation and wormhole models (see, e.g., Refs. [14,15]).

In both examples, a physical boundary separates two regions with different values of $\Lambda$ and serves as the interface between two vacua with different values of the vacuum energy. The problem of coexisting vacua in general relativity and elementary particle physics is rapidly becoming mainstream, not without the influence of condensed-matter physics.

Reference [16], for example, considers a Friedmann-Robertson-Walker island embedded in a de-Sitter spacetime with nonzero cosmological constant. For elementary particle physics, the so-called Multiple Point Principle has been introduced [17], according to which Nature chooses the parameters of the Standard Model so that two or more phases of quantum vacua have the same energy density and can coexist. Coexistence of quantum vacua has also been discussed in relation to the cosmological constant problem [18], where it was 
found that the equilibrium value of $\Lambda$ in the exterior region vanishes (based on an analog from condensed-matter physics and a thermodynamic argument applied to the vacuum of relativistic quantum fields).

Furthermore, there may exist bubbles of false vacuum, whose stability is provided by the conservation of a fermionic charge $Q$ trapped within the bubble or on the interface. These analogs of $Q$-balls [19] have been discussed in Ref. [20] (see also references therein). Regarding the nature of the fermionic charge $Q$, possible examples are the baryon charge $B$, the lepton charge $L$, or the difference $B-L$, which is exactly conserved in the Standard Model whereas $B+L$ is not [21].

As a particularly simple case, we discuss in the next section the embedded de-Sitter Universe and one possible type of interface. In the final section, we present some concluding remarks.

\section{De-Sitter bubble}

\subsection{Pressure equilibrium}

Let us consider the de-Sitter-Schwarzschild bubble from Eqs. (4ab) using thin-wall techniques (see $[14,15]$ and references therein). The interface (membrane) at radius $r=R$ could be a topological domain wall between two almost-degenerate vacuum states. This wall is assumed to be very thin compared to the characteristic size of the Universe, $\xi \ll R$, and can be considered as a delta-function singularity in the energy-momentum tensor for the matter fields.

The detailed structure within the domain wall need not be considered, since the general properties of the domain wall can be described in terms of the $(2+1)$-dimensional energy-momentum tensor of the membrane, which is characterized by two parameters, the surface energy density $\epsilon$ and the surface tension $\sigma$. In the thin-wall approach, the jump of the metric across the interface can be expressed in terms of the surface energy density $\epsilon$ of the interface:

$$
\epsilon=\frac{1}{4 \pi G R}\left[\sqrt{1-R^{2} / R_{0}^{2}}-\sqrt{1-2 G M / R}\right] .
$$

The equilibrium radius of the bubble can be found from the thermodynamic identity which expresses the two-dimensional pressure $p$ of the interface (or the surface tension $\sigma=-p$ ) through the surface energy,

$$
p=-\sigma=-\frac{d(\epsilon A)}{d A}=-\frac{1}{2 R} \frac{d\left(\epsilon R^{2}\right)}{d R}
$$


where $A \equiv 4 \pi R^{2}$ is the area of the interface. If the energy density $\epsilon$ does not depend on $R$, one has simply $p=-\epsilon$.

Taking the derivative of Eq. (6) and using Eq. (7), one obtains

$$
p=\frac{1}{8 \pi G R}\left[\frac{1-G M / R}{\sqrt{1-2 G M / R}}-\frac{1-2 R^{2} / R_{0}^{2}}{\sqrt{1-R^{2} / R_{0}^{2}}}\right] .
$$

Provided the surface energy $4 \pi R^{2} \epsilon(R)$ is known, Eqs. (6)-(8) determine the

radius $R$ and mass $M$ of the equilibrium bubble as functions of $R_{0}$ (or of $\left.\Lambda_{0} \equiv 3 / 8 \pi G R_{0}^{2}\right)$.

\subsection{Stabilization by fermion zero modes}

For the case of fermionic charge concentrated at the interface, the surface energy density $\epsilon$ clearly depends on $R$. Let us suppose that the interface contains fermion zero modes, that is, massless $(2+1)$-dimensional relativistic fermions with spectrum $E(\mathbf{p})=c|\mathbf{p}|$. If the chemical potential $\mu$ of these fermions is nonzero, the energy density of the interface includes a contribution from the fermions,

$$
\epsilon=\epsilon^{0}+\frac{\mu^{3}}{6 \pi}
$$

The surface tension is then given by

$$
\sigma=-p=\epsilon-\mu n=\epsilon-\frac{\mu^{3}}{4 \pi}=\epsilon^{0}-\frac{\mu^{3}}{12 \pi}
$$

where $n=\mu^{2} / 4 \pi$ is the number density of $(2+1)$-dimensional fermions. (See Ref. [22] for a different mechanism to stabilize the interface using $(2+1)-$ dimensional gauge fields.)

The same surface tension can be obtained from Eq. (7) if one writes the fermionic contribution to surface energy density in Eq. (9) in terms of the bubble radius for a fixed number of fermions, $N=4 \pi R^{2} n=R^{2} \mu^{2}$ :

$$
\epsilon(R)=\epsilon^{0}+\frac{N^{3 / 2}}{6 \pi R^{3}}, \quad \sigma(R)=\epsilon^{0}-\frac{N^{3 / 2}}{12 \pi R^{3}} .
$$

This dependence of the surface energy density allows us to regulate the bubble radius by changing the total fermionic charge $N$ of the interface. With the fermion charge concentrated on the surface, this object could perhaps be called a $Q$-shell, but we will adhere to the more general terminology of $Q$-ball. 


\subsection{Weakly gravitating Q-ball}

Let us first discuss the case $R \ll R_{0}$. The Universe then occupies only a small fraction of the volume of the cosmological horizon and the effect of gravity is weak and can be ignored. In this limit, we have a non-gravitating false-vacuum bubble without matter. Its energy is given by

$$
E=M=\frac{4 \pi}{3} R^{3} \Lambda_{0}+4 \pi R^{2} \epsilon
$$

which also follows from Eq. (6) for $R \ll R_{0}$.

Using the surface energy density from Eq. (11), one obtains the energy of the de-Sitter bubble at fixed $N$ :

$$
E(R, N)=\frac{4 \pi}{3} R^{3} \Lambda_{0}+4 \pi R^{2} \epsilon^{0}+\frac{2}{3 R} N^{3 / 2} .
$$

The equilibrium condition $d E / d R=0$ corresponds to pressure balance and gives the vacuum pressure inside the bubble, $P_{\text {vac }} \equiv-\Lambda_{0}$, in terms of the surface tension $\sigma=-p$ :

$$
P_{\mathrm{vac}} \equiv-\Lambda_{0}=\frac{2 \sigma(R)}{R}
$$

This last equation takes into account that the vacuum pressure outside the bubble is zero, $P_{\text {vac }} \equiv-\Lambda=0$. Nullification of the cosmological constant in the exterior region may be a consequence of thermodynamic stability for a system isolated from its environment [18].

Equation (14) gives the radius of the $Q$-ball in terms of $\Lambda_{0}$. Since $\Lambda_{0}>0$, the equilibrium false-vacuum $Q$-ball exists only for negative surface tension, which, according to Eq. (10) or (11), is realized for large enough chemical potential $\mu$ of trapped fermions. The condition for ignoring gravity is $R \ll R_{0}$, which holds for $\sigma^{2} \ll \Lambda_{0} / G$.

For the corresponding weakly gravitating Einstein bubble (2ab), the radius $R$ is determined by a pressure-balance equation similar to Eq. (14). Now, the total internal pressure is compensated by surface tension:

$$
P_{\mathrm{vac}}+P_{\mathrm{m}} \equiv-\Lambda_{0}+w \rho_{\mathrm{m}}=\frac{2 \sigma(R)}{R}
$$

for matter equation of state $P_{\mathrm{m}}=w \rho_{\mathrm{m}}$.

\subsection{Asymptotic horizons}

Let us return to the gravitating de-Sitter $Q$-ball ( $Q$-shell) and find the conditions under which $R$ can asymptotically approach the cosmological horizon 
$R_{0}$. Analysis of Eqs. (6)-(8) shows that this occurs for negative surface tension $\sigma$ under the following conditions ${ }^{1}$ :

$$
\epsilon \ll \sqrt{\Lambda_{0} / G} \ll|\sigma|
$$

Under these conditions, one has

$$
1-2 G M / R \approx 1-R^{2} / R_{0}^{2} \approx \frac{3}{32 \pi} \frac{\Lambda_{0}}{G \sigma^{2}} \ll 1
$$

In the limit of large $|\sigma|$, a cosmological horizon is developing asymptotically from the inside and a black-hole horizon from the outside. For a 'poor physicist' living inside the bubble, the interior of the bubble resembles more and more a de-Sitter Universe, while, for another 'poor physicist' living outside, the exterior of the bubble resembles more and more a black hole. And the smooth interface between the two vacua (with nonsingular metric) starts to look like a genuine coordinate singularity. In the same way, the finite and curved Einstein Universe may be thought of as the limiting case of a false-vacuum bubble (2ab) in flat space.

However, the limiting case of a de-Sitter horizon cannot be realized with an interface of fermion zero modes as discussed in Sec. 2.2. The reason is that Eqs. (11) and (16) require the energy of the interface without fermion-zeromode contribution to be negative, $\epsilon^{0}<0$. Hence, the static de-Sitter Universe with cosmological horizon probably cannot be constructed using known interfaces, even though suitable Planck-scale interfaces are not excluded.

\section{Discussion}

The static de-Sitter Universe [8] (or the static Einstein Universe [6]) and the $Q$-ball with false vacuum $[19,20]$ can be viewed as extreme limits of the same physical object. For both, the vacuum energy is of crucial importance, as well as the surface energy density and (negative) surface tension of the membrane separating the interior region from the external environment. In addition, there may be an important role for a conserved fermionic charge $Q$ inside the bubble or on the membrane.

In the limit of small surface energy density and large negative surface tension, a cosmological horizon is developing asymptotically from the inside and

1 The approximation of having surface energy density $\epsilon$ and tension $\sigma$ as quantities independent of gravity is only valid if the relative jump of the metric is small. This gives the following refinement of conditions (16): $\epsilon \ll \Lambda_{0} / G|\sigma| \ll \sqrt{\Lambda_{0} / G} \ll|\sigma|$. 
a black-hole horizon from the outside. In this way, one nearly obtains a static de-Sitter Universe embedded in a Schwarzschild spacetime as discussed previously [11-13] for black holes with physical horizons and false vacua in their interior. However, the limiting case cannot be realized using known physical interfaces.

This also implies that, most likely, emergent gravity is not able to incorporate the geodesically-complete Einstein Universe with spatial section $S^{3}$. (For a Hausdorff manifold, this would indeed be difficult to imagine topologically.) It, therefore, appears that the original static $S^{3}$ Einstein Universe can exist only within the context of fundamental general relativity.

\section{Acknowledgements}

F.R.K. thanks M.J.G. Veltman for valuable discussions over the years. The work of G.E.V. is supported in part by the Russian Ministry of Education and Science, through the Leading Scientific School grant \#2338.2003.2. This work is also supported by the European Science Foundation COSLAB Program.

\section{References}

[1] G.E. Volovik, The Universe in a Helium Droplet (Clarendon Press, Oxford, 2003).

[2] B.L. Hu, "Can spacetime be a condensate?" gr-qc/0503067.

[3] C.D. Froggatt and H.B. Nielsen, "Derivation of Poincaré invariance from general quantum field theory," Ann. Phys. (Leipzig), 14, 115 (2005), hep-th/0501149.

[4] M.J.G. Veltman, "Quantum theory of gravitation," in Methods in Field Theory, Les Houches 1975, eds. R. Balian and J. Zinn-Justin (North-Holland, Amsterdam, 1976).

[5] F.R. Klinkhamer and M.J.G. Veltman, "Gravity without curved space," unpublished notes, 1992.

[6] A. Einstein, 'Kosmologische Betrachtungen zur allgemeinen Relativitätstheorie," Sitzungsber. Preuss. Akad. Wiss. 1917, p. 142; translated in H.A. Lorentz et al., The Principle of Relativity (Dover Publ., New York, 1952).

[7] C. Mфller, The Theory of Relativity (Clarendon Press, Oxford, 1952).

[8] W. de Sitter, "On the relativity of inertia. Remarks concerning Einstein's latest hypothesis," Proc. Kon. Acad. Amst. 19, 1217 (1917); "On the curvature of space," ibid. 20, 229 (1917). 
[9] H. Weyl, "Über die statischen kugelsymmetrischen Lösungen von Einsteins 'kosmologischen' Gravitationsgleichungen," Phys. Z. 20, 31 (1919).

[10] R.B. Laughlin, "Emergent relativity," Int. J. Mod. Phys. A18, 831 (2003).

[11] G. Chapline, E. Hohlfeld, R.B. Laughlin, and D.I. Santiago, "Quantum phase transitions and the breakdown of classical general relativity," Phil. Mag. B 81, 235 (2001), gr-qc/0012094v1.

[12] P.O. Mazur and E. Mottola, "Gravitational vacuum condensate stars," Proc. Nat. Acad. Sci. 111, 9545 (2004), gr-qc/0407075.

[13] I. Dymnikova and E. Galaktionov, "Stability of a vacuum nonsingular black hole," gr-qc/0409049.

[14] S.K. Blau, E.I. Guendelman, and A.H. Guth, "Dynamics of false-vacuum bubbles", Phys. Rev. D 35, 1747 (1987).

[15] M. Visser, "Traversable wormholes: some simple examples," Phys. Rev. D 39, 3182 (1989); M. Visser, Lorentzian Wormholes: From Einstein to Hawking (Springer, New York, 1996), Chaps. 14 and 15.

[16] S. Dutta and T. Vachaspati, "Islands in the $\Lambda$-sea," Phys. Rev. D 71, 083507 (2005), astro-ph/0501396.

[17] D.L. Bennett and H.B. Nielsen, "Predictions for non-Abelian fine structure constants from multicriticality," Int. J. Mod. Phys. A 9, 5155 (1994), hep$\mathrm{ph} / 9311321$.

[18] G.E. Volovik, "Coexistence of different vacua in the effective quantum field theory and multiple point principle," JETP Lett. 79, 101 (2004), hepph/0309144; G.E. Volovik, "On contributions of fundamental particles to the vacuum energy," in: I.Ya. Pomeranchuk and Physics at the Turn of the Century, eds. A. Berkov, N. Narozhny, and L. Okun (World Scientific, Singapore, 2003) p. 234, hep-ph/0306011.

[19] S. Coleman, "Q balls," Nucl. Phys. B 262, 263 (1985).

[20] R.G. Daghigh and Y. Hosotani, "Gravitating fermionic lumps with a false vacuum core," Prog. Theor. Phys. 110, 1151 (2004), gr-qc/0307075.

[21] G. 't Hooft, "Symmetry breaking through Bell-Jackiw anomalies," Phys. Rev. Lett. 37, 8 (1976); N.H. Christ, "Conservation law violation at high-energy by anomalies," Phys. Rev. D 21, 1591 (1980); F.R. Klinkhamer and Y.J. Lee, "Spectral flow of chiral fermions in nondissipative gauge field backgrounds," Phys. Rev. D 64, 065024 (2001), hep-th/0104096.

[22] E.I. Guendelman and J. Portnoy, "The universe out of an elementary particle?" Class. Quant. Grav. 16, 3315 (1999), gr-qc/9901066. 